\title{
NORMAS INDEPENDIENTES, CRITERIOS CONCEPTUALES Y TRUCOS VERBALES. RESPUESTA A EUGENIO BULYGIN
}

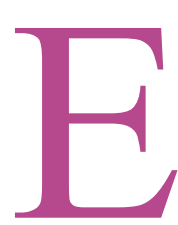

n el artículo Algunas consideraciones sobre los sistemas jurídicos, Eugenio Bulygin ha tenido conmigo la atención de dirigir algunas críticas a ciertas tesis defendidas en mi libro Jurisdicción y normas. Para quien conozca este libro, de más estará decir que Eugenio Bulygin, es uno de los filósofos del Derecho de quienes más he aprendido: la deuda intelectual que tengo con él y con Carlos Alchourrón aparece explícitamente reconocida un sinnúmero de veces a lo largo del texto. $\mathrm{Y}$ ya en la nota preliminar advierto que la lectura que E. Bulygin hizo de su primera versión me permitió eliminar de la versión definitiva algún error no menor en el que había incurrido respecto del problema de las lagunas normativas.

Dos son los puntos en los que se centra la crítica de Bulygin: (1) la crítica dirigida por mí a su propuesta -contenida en el artículo de 1976 Sobre la regla de reconocimientode entender la regla de reconocimiento como una regla o criterio conceptual y no como una norma de conducta y mi defensa de esta última alternativa; (2) mi propuesta de caracterizar el concepto de «juez» extrasistemáticamente, por referencia a reglas sociales aceptadas y no a reglas jurídicas válidas, Me ocuparé de cada una de ellas por separado, aunque, como es obvio, se trata de problemas no del todo desconectados.

\section{1}

1.1. En Jurisdicción y normas escribí que «en la caracterización hecha por Bulygin de la regla de reconocimiento y en su consideración de la misma como regla conceptual hay una confusión entre la propia regla de reconocimiento (como norma o regla de conducta aceptada por los jueces) y el criterio de identificación 
de las reglas del sistema basado en la regla de reconocimiento (como criterio usado por un observador externo, como pueda ser, por ejemplo, un teórico del Derecho o un consejero jurídico privado)» (Ruiz Manero, 1990, pp. 137-138). Este cargo le parece a Bulygin «enteramente injusto» y señala que «en ningún momento confundí la regla de reconocimiento como norma de conducta con el criterio de identificación de las reglas del sistema basado en tal regla de reconocimiento. Lo que hice era muy distinto: propuse llamar «regla de reconocimiento» al criterio de identificación» (Bulygin, 1991).

Debo decir que el uso, por mi parte, del término «confusión» fue muy probablemente desafortunado, porque podría entenderse en el sentido de imputar a Bulygin un desdibujamiento de la distinción entre una norma de conducta y una regla o criterio conceptual. Entendido así, hablar de «confusión»a propósito del texto de Bulygin de 1976 es, sin duda, enteramente injusto: precisamente buena parte del artículo Sobre la regla de reconocimiento se dedica a trazar nítida e impecablemente la distinción entre norma de conducta y regla conceptual. Mi crítica tenía otro alcance: lo que yo sostenía era que el criterio o regla conceptual empleado por los teóricos del Derecho para el fin cognoscitivo de determinar los límites del sistema jurídico presupone la regla de reconocimiento como norma de conducta aceptada por la judicatura. Y ello en el sentido de que el enunciado formulado por un teórico del Derecho y referido a una norma distinta de la regla de reconocimiento, según el cual "la norma n pertenece al sistema jurídico S", es verdadero si, y sólo si, la norma $\mathrm{n}$ es conforme con los criterios de validez jurídica aceptados como vinculantes por la judicatura del sistema $\mathrm{S}$ (y es al conjunto de criterios de validez jurídica aceptados por dicha judicatura a lo que denominados la regla de reconocimiento del sistema $\mathrm{S})$. Dicho en otros términos, una cosa -sostenía yo entonces y quiero seguir defendiendo ahora- es la norma última del sistema que prescribe a sus órganos de aplicación aplicar las normas que satisfagan determinados criterios y otra cosa -y parasitaria de la anterior- es el criterio conceptual basado en una proposición normativa que describe cuáles son los criterios de validez jurídica contenidos en dicha norma última. Dicho aún de otra forma, y utilizando una distinción a la que apela el propio Bulygin en su respuesta a mi crítica: los jueces usan unos criterios de validez jurídica aceptados por el conjunto de ellos con la finalidad práctica de identificar a las normas que tienen el deber de aplicar; los teóricos del Derecho mencionan estos criterios de validez para la finalidad teórica de determinar las normas 
integrantes del sistema jurídico y consideran como tales a las que satisfacen los criterios de validez aceptados y usados por el conjunto de la judicatura.

E. Bulygin sintetiza ahora sus diferencias con la posición hartiana (con la que yo muestro mi acuerdo en el libro) de la siguiente forma: «ellos creen que primero tenemos una norma que obliga a aplicar las normas que reúnen ciertas características y luego, de esta norma se extrae el criterio conceptual que define qué normas pertenecen al sistema; yo, en cambio, sostengo que para poder determinar qué deberes establecen las normas jurídicas, hay que tener primero un criterio conceptual que nos permita identificar tales normas». Creo que esta afirmación es susceptible de la misma crítica que formulé en Jurisdicción y normas a propósito del artículo de 1976. Pues me parece que Bulygin sigue aquí sin diferenciar adecuadamente dos problemas distintos: el primero es el del fundamento normativo del deber de aplicar las normas (o clases de normas) que reúnan determinadas características; el segundo es el de las condiciones de verdad de los enunciados que afirman la existencia de tal deber respecto de una determinada norma o clase de normas (y, consiguientemente, la pertenencia de tal norma o clase de normas al sistema jurídico). El primer problema se encuentra situado en el plano de esa institución social normativa a la que llamamos sistema jurídico; el segundo en el plano de la descripción de esa institución social normativa.

1.2. Aunque pienso que lo anterior es correcto, no me parece, sin embargo, que seguir argumentando con arreglo a los lineamientos precedentes tenga demasiada utilidad: con ello no haría sino mostrar que mi desacuerdo con el Bulygin de 1976 permanece idéntico con el Bulygin de 1991. Reiterar una vez más los argumentos que dirigí contra el artículo de Bulygin de 1976 sería enteramente gratuito: por lo que hace a los eventuales lectores interesados en esta polémica, dichos argumentos se encuentran en Jurisdicción y normas y por lo que hace a E. Bulygin, no me parece que la reiteración de los mismos argumentos vaya a impresionarle mucho más que su primera enunciación.

Procederé, por ello, a discutir la tesis de Bulygin según la cual «la unidad del orden jurídico y de cada uno de los sistemas que pertenecen a él está constituida por una regla conceptual (criterio de identificación) y no por una norma de conducta», atendiendo a cómo aparece desarrollada esta tesis en el artículo de 1991. Partiré, para ello, del «criterio de identificación de un orden jurídico determinado $\mathrm{O}_{1}$ » propuesto por el propio Bulygin y que consta de cinco reglas (o subreglas) conceptuales: 
$\mathrm{O}_{1}$.

«(1) El conjunto de normas $\left(\mathrm{N}_{1}, \mathrm{~N}_{2} \ldots \mathrm{N}_{\mathrm{n})}\right.$ es el sistema originario (primera constitución) de

(2) Si una norma $N_{j}$ es válida en un sistema $S_{t}$, que pertenece a $\mathrm{O}_{1}, y \mathrm{~N}_{\mathrm{j}}$ faculta a la autoridad $x$ a promulgar la norma $\mathrm{N}_{\mathrm{k}}$ y x promulga en el momento t la norma $\mathrm{N}_{\mathrm{k}}$, entonces $\mathrm{N}_{\mathrm{k}}$ es válida en el sistema $\mathrm{S}_{\mathrm{t}+1}$ pertenece a $\mathrm{O}_{1}$.

(3) Si una norma $\mathrm{N}_{\mathrm{j}}$ es válida en un sistema $\mathrm{S}_{\mathrm{t}}$, que pertenece a $\mathrm{O}_{1}, \mathrm{y} \mathrm{N}_{\mathrm{j}}$ faculta a la autoridad $x$ a derogar la norma $N_{k}$ que es válida en $S_{t}$ y x deroga $N_{k}$ en el momento t, entonces $N_{k}$ no es válida en el sistema $S_{t+1}$, (correspondiente al momento siguiente a t) que pertenece a $\mathrm{O}_{1}$.

(4) Las normas válidas en el sistema $\mathrm{S}_{\mathrm{t}}$, que pertenece a $\mathrm{O}_{1}$, que no han sido derogadas en el momento $t$, son válidas en el sistema $\mathrm{S}_{\mathrm{t}}$ de $\mathrm{O}_{1}$, (que corresponde al momento siguiente a $\mathrm{t}$ ).

(5) Todas las consecuencias lógicas de las normas válidas en un sistema $S_{t}$, que pertenece a $\mathrm{O}_{1}$, también son válidas en $\mathrm{S}_{\mathrm{t}}$.»

Como añade el propio Bulygin, «los criterios de pertenencia de una norma a un sistema son tres: a) criterio de pertenencia directa de las normas independientes [esto es, de las normas situadas en el nivel de la regla (1) del criterio de identificación]; b) criterio de deducibilidad; c) criterio de legalidad».

De acuerdo con ello, en el sistema jurídico hay, por un lado, normas no independientes, esto es normas que pertenecen al sistema en virtud de su relación (de deducibilidad o de legalidad) con otras normas y, por otro lado, normas independientes (normas que pertenecen directamente al sistema). Dejaré de lado, por escasamente relevante, el caso de las normas que constituyen meras consecuencias lógicas de otras normas (pues, dicho sea de paso, no entiendo en qué sentido puede decirse que una consecuencia lógica de una norma sea otra norma si, por definición, no puede contener nada que no se encuentre contenido en la primera) y me centraré en las normas no independientes que pertenecen al sistema en virtud de su relación de legalidad con otras normas y en las normas independientes.

Por lo que hace a las normas no independientes que pertenecen al sistema en virtud de su relación de legalidad con otras normas, parece que un enunciado del tipo "la norma $\mathrm{N}$ pertenece al sistema porque ha sido promulgada por una autoridad facultada para ello por la norma $\mathrm{N}_{1}$ " es verdadero si, y sólo si, la norma $\mathrm{N}$ ha sido promulgada por una autoridad facultada para ello por la norma $\mathrm{N}_{1}$. $\mathrm{O}$, dicho de otra forma, un enunciado del tipo "la norma $\mathrm{N}_{1}$ faculta a la autoridad $\mathrm{x}$ para promulgar la norma $\mathrm{N}$ " es verdadero si, y sólo si, corresponde al contenido de la norma $\mathrm{N}_{1}$ esto es si, y sólo si, la norma $\mathrm{N}_{1}$ faculta a la autoridad 
x para promulgar la norma N. En el caso, pues, de estas normas no independientes, los enunciados que afirman su pertenencia tienen como condición necesaria de su verdad la correspondencia entre tales enunciados y el contenido de otras normas.

Supongamos, ahora, que $\mathrm{N}_{1}$ sea una norma independiente. El enunciado que afirma que " $\mathrm{N}_{1}$ pertenece directamente al orden jurídico $\mathrm{O}_{1}$ ” ¿está sujeto a condiciones de verdad o debe ser considerado, por el contrario, como un axioma? Me parece que la respuesta que se dé a esta pregunta es decisiva para validar o invalidar lo que viene a ser el núcleo de la posición de Bulygin: a saber, la prioridad del criterio de identificación sobre cualesquiera normas. Y me parece también que la respuesta que se dé a esta pregunta depende de si lo que tratamos de identificar son órdenes jurídicos reales, o, si, por el contrario, no nos importa discriminar entre tales órdenes jurídicos y otros órdenes jurídicos, imaginados, propuestos o añorados. Veamos una y otra cosa.

Digo que esta cuestión es decisiva para la posición de Bulygin porque, como él mismo señala, la identidad de un orden jurídico depende de la identidad de sus normas independientes. Escribe el propio Bulygin que la regla (1) de su criterio de identificación (la que señala la pertenencia directa de ciertas normas al sistema) «es la que identifica al orden considerado; otros órdenes se distinguen de $\mathrm{O}_{1}$ no por su estructura (que es idéntica), sino por ser distinta su primera constitución, es decir, por la regla (1)». Pues bien, la identificación de las normas independientes de un determinado orden ¿debe considerarse como un axioma o como un enunciado susceptible de ser calificado como verdadero o falso? Si fuera el caso de lo primero, Bulygin tendría razón en su consideración de que el criterio de identificación tiene prioridad sobre cualesquiera normas; pero si fuera el caso de lo segundo lo prioritario sería el que determinadas normas fueran de hecho o no lo fueran las normas independientes del orden de que se tratara; los enunciados constitutivos de la regla (1) serían verdaderos (o falsos) según que correspondieran (o no) al hecho de que las normas en ellos mencionadas fueran, en efecto, las normas independientes del sistema considerado.

Que el considerar como axiomas a los enunciados que afirman que determinadas normas son las normas independientes de un determinado orden jurídico sólo puede hacerse al precio de renunciar a distinguir entre órdenes jurídicos reales y órdenes jurídicos imaginados, añorados o propuestos es, me parece, algo que el propio Bulygin implícitamente admite. Bulygin, en efecto, 
tras escribir que «cualquier conjunto de normas (que contenga al menos una norma de competencia) puede ocupar la posición de una primera constitución y dar lugar a un orden jurídico» añade que "por supuesto, no todos los órdenes jurídicos son igualmente interesantes; a veces los juristas están interesados en aquel orden jurídico que es efectivo o vigente en una sociedad dada en un tiempo determinado. Pero éste es otro problema». Y en el ámbito de este «otro problema» -la identificación del orden jurídico efectivo o vigente en una sociedad dada en un tiempo determinado- hay que situar, me parece, las referencias de Bulygin a diversas «revoluciones jurídicas»-la revolución rusa de 1917, la promulgación de la Constitución española de 1978, la restauración de la Constitución argentina en 1983como constitutivas de otras tantas rupturas de órdenes jurídicos y orígenes de nuevos órdenes.

Enunciados tales como (i) «en 1932 la Constitución monárquica de 1876 pertenecía directamente al orden jurídico vigente en España» y (ii) «en 1932 la Constitución republicana de 1931 pertenecía directamente al orden jurídico vigente en España» no parece que puedan ser interpretados razonablemente como axiomas irrefutables, sino como proposiciones que dicen algo acerca del mundo, que tratan de identificar normas independientes pertenecientes al orden jurídico que, en efecto, estaba en vigor en España en 1932, y que, como tales, son verdaderas o falsas. Y en este sentido, concordará todo el mundo en que (i) es falso en tanto que (ii) es verdadero. Y también concordará todo el mundo en que sólo a partir de (ii) podemos construir un criterio de identificación de las normas que pertenecían al sistema jurídico vigente en España en 1932 que identifique verdaderamente a las normas que, en efecto, pertenecían a tal sistema jurídico. Y ello es así porque en 1932 los órganos de aplicación españoles -los jueces y tribunales, en sentido amplio- reconocían en su conjunto como vinculantes para ellos la Constitución republicana de 1931 y las normas dictadas o recibidas de acuerdo con ella y no reconocían como tales a la Constitución monárquica de 1876 y a las normas dictadas o recibidas de acuerdo con ella. En definitiva, porque la regla de reconocimiento aceptada por los órganos de aplicación reconocía a la Constitución republicana de 1931 y no a la Constitución monárquica de 1876 como la norma suprema del sistema.

Alguien podría objetar que para considerar así las cosas no es preciso acudir a una regla de reconocimiento entendida como una práctica normativa última de carácter consuetudinario, sino que basta con el expediente, aparentemente más simple, de la 
eficacia general». Se dirá, así, que en la España de 1932 la Constitución de 1931 y las normas dictadas o recibidas de acuerdo con ella eran «generalmente eficaces», lo que no ocurría con la Constitución monárquica de 1876. Pero a esto cabe replicar que la «eficacia general» de las normas jurídicas generales consiste, al menos en una parte sustancial, en que el contenido de las normas individuales dictadas por los órganos de aplicación corresponda al contenido de dichas normas generales. Pero ocurre además, que no siempre el criterio de la «eficacia general» así entendida -como correspondencia entre el contenido de las normas generales y el de las normas individuales dictadas por los órganos de aplicación- es suficiente para discriminar la verdad o falsedad de los enunciados que pretenden identificar normas pertenecientes a un orden jurídico. Supongamos, reformulando un ejemplo de J. Raz (1990), que un grupo de profesores de Derecho hubiera propuesto en enero de 1932 una reforma constitucional cuyo texto resultante sólo difiriera del de la Constitución de 1931 en algunos detalles no centrales y estableciera, a grandes rasgos, un sistema de fuentes prácticamente igual al establecido en dicha Constitución. Si atendiéramos meramente a la «eficacia general» en el sentido indicado de correspondencia, bien pudiera ocurrir que no fuera posible determinar si la Constitución vigente en España en febrero de 1932 era la Constitución republicana de 1931 o la «Constitución de los profesores», pues el grado de correspondencia entre una y otra, de un lado, y la conducta normativa de los órganos de aplicación, de otro, fuera muy semejante. Lo que permite determinar tal cosa es el hecho de que la judicatura en su conjunto consideraba vinculante para ella a la Constitución del 31 y no a la «Constitución de los profesores». O, dicho de otra forma, el hecho de que la judicatura en su conjunto aceptaba una regla última en virtud de la cual era la Constitución del 31, y no la «Constitución de los profesores», la norma suprema del sistema [la importancia de la distinción hartiana entre norma última y norma suprema para comprender adecuadamente la relación entre la regla de reconocimiento de un sistema y la norma de aquélla señala como norma suprema del mismo sistema ha sido excelentemente destacada, en un artículo todavía reciente, por A. Ruiz Miguel (1988)].

2

2.1. Escribe Bulygin, con cierto énfasis, que «para justificar una acción no es suficiente mencionar una norma, hay que usarla». 
Esto es sin duda cierto, pero deja intacto el problema de la justificación de la decisión de usar una determinada norma. A este respecto, Bulygin parece dar a entender que la decisión de usar una norma no requiere ulterior justificación y, así, escribe que «si uno quiere saber qué debe hacer, tiene que usar una norma y no preguntar por su fundamento». Pero resulta verdaderamente difícil de comprender por qué, frente a la decisión de usar una norma, habría de resultar impertinente la pregunta por la justificación de esa decisión, pregunta perfectamente pertinente a propósito de cualquier otra decisión. No se alcanza a entender por qué es pertinente preguntar a un juez cuál fue la razón por la que condenó a un individuo $\mathrm{x}$ a una determinada pena $\mathrm{y}$, sin embargo, frente a su respuesta de que lo hizo porque así lo ordenaba la norma $\mathrm{N}$, que usó como fundamento de su decisión, ya no es pertinente preguntar cuál fue la razón por la que usó la norma $\mathrm{N}$ como fundamento de su decisión. Quizá alguien pudiera desear sostener que la posición de Bulygin no es que la decisión de usar una norma no requiera justificación, sino que, por lo que hace al Derecho, tal justificación viene aportada por el criterio conceptual con arreglo al cual identificamos ciertas normas como jurídicas. Quien deseara entender así la posición de Bulygin podría, quizá, aducir en su favor el que éste escribe que «si se trata de un juez, debe usar las normas jurídicas y para identificarlas tiene que recurrir a un criterio conceptual, es decir, a una definición» (el subrayado es mío). Esto -argüiría quien así interpretara a Bulygin- habría que entenderlo de la siguiente forma: Bulygin admite que el juez tiene el deber de usar las normas jurídicas, y no cualesquiera otras normas, como fundamento de sus decisiones. Admitido esto, Bulygin no puede eludir la pregunta por el fundamento de este deber y su respuesta a la misma sería que los jueces tienen tal deber en virtud de «un criterio de identificación compartido» con arreglo al cual se identifican las normas jurídicas. Pero si esta interpretación fuera correcta -esto es, si expresara adecuadamente lo que Bulygin quiere decir- la posición de Bulygin sería aún más difícil de entender, pues no se alcanza a ver cómo alguien que no desee caer de lleno bajo la guillotina de Hume puede considerar que un criterio conceptual -esto es, un enunciado teórico- puede constituir el fundamento de deber alguno.

2.2. Pero el caso es que el propio Bulygin, pese a sus protestas en contrario, también alude en alguna ocasión al deber de aplicar ciertas normas como fundamentado en otras normas. Así, por ejemplo, cuando dice que el juez, para determinar las normas a usar como fundamento de su decisión, «debe usar los 
criterios de aplicabilidad vigentes», parece aludir a criterios de aplicabilidad contenidos en normas jurídicas que determinan qué otras normas jurídicas tiene el juez el deber de aplicar. Así lo sugiere el que como ejemplo de tales criterios de aplicabilidad ponga «la regla de la ley más benigna en Derecho penal [en virtud de la cual] el juez debe aplicar la ley penal más benigna». Lo que ocurre es que Bulygin, en relación con la pregunta por el fundamento del deber de usar una determinada norma, se detiene ante las normas independientes del orden jurídico, ante aquellas normas que vienen señaladas directamente por la regla de reconocimiento aceptada. Es el uso de estas normas el que, en su opinión, o bien no requeriría justificación, o bien -de ser correcta esta segunda interpretación- se justificaría mediante el criterio conceptual.

Bulygin se pregunta si «acaso los jueces no pueden preguntar por qué deben obedecer la regla de reconocimiento» y señala que «no se ve por qué la mayoría de los filósofos del Derecho de repente se paran frente a ciertas respuestas y no siguen preguntando». Y entre dicha mayoría parece sin ninguna duda incluirme, pues advierte que mi consideración de la regla de reconocimiento como norma jurídicamente última «equivale a la respuesta del jefe: “¡No pregunte más, haga lo que se le ha ordenado!”». Podría yo replicar, y creo que con razón, que es su posición -jidentifique las normas jurídicas mediante un criterio conceptual, úselas para fundamentar sus decisiones y no se haga una sola pregunta más!- la que equivale exactamente a "a respuesta del jefe": «No pregunte más, haga lo que se [el Derecho] le ha ordenado!».

Pero lo que me importa no es devolverle el reproche a Bulygin, sino subrayar que mi consideración de la regla de reconocimiento como una normal jurídicamente última no tiene nada que ver con "la respuesta del jefe", pues no implica en modo alguno situar en dicha norma un término final a la pregunta por el fundamento del deber de obediencia. Que la regla de reconocimiento será jurídicamente última quiere decir, simplemente, que a la pregunta por el fundamento del deber de obedecerla no puede darse ya ninguna respuesta jurídica, no que no pueda darse ninguna respuesta. Lo que sostengo en Jurisdicción y Normas (cap. segundo, III, 4, págs. 166-179) -y no soy en ello, desde luego, nada original, sino que sigo las huellas de autores como Raz o Nino- es que al ser la regla de reconocimiento una norma que ordena a los jueces usar otras normas como fundamento de sus decisiones, y al imponer estas normas deberes a personas distintas de los propios jueces, la aceptación de la regla de reconocimiento 
por parte de los jueces sólo puede justificarse por razones morales. Bulygin, haciendo referencia a Nino, señala que esto no ofrece una respuesta satisfactoria, «porque también cabe preguntar "¿Por qué debo obedecer las reglas morales?”». A esto sólo puedo contestar, con palabras del mismo Nino, que «por convención llamamos "morales" a las razones [justificatorias] operativas de mayor jerarquía» (Nino, 1985, pág. 133), que «las razones de índole moral excluyen cualquier otro tipo de razones en las situaciones en que son aplicables» y que ello constituye una «regla de prioridad» que impide la desintegración de nuestro razonamiento práctico (ibid., pág. 65).

Bulygin se muestra de acuerdo conmigo en que la teoría de Hart es circular en el sentido de que con arreglo a ella, para poder determinar, de un lado, el contenido de la regla de reconocimiento de un sistema jurídico hemos de determinar previamente quiénes son jueces de dicho sistema, lo que sólo podemos hacer sobre la base de reglas de adjudicación del propio sistema $\mathrm{y}$, de otro lado, para poder determinar cuáles son las reglas de adjudicación del sistema hemos de acudir a su regla de reconocimiento. Por ello quienes, aceptando el marco conceptual hartiano, hemos tratado de romper esta circularidad -como Carlos Nino (1979 y 1980), Nell MacCormick (1981) y yo mismo (1990)- coincidimos en proponer caracterizaciones del concepto de «juez» que sean, respecto del sistema jurídico (entendido como compuesto por la regla de reconocimiento y por las normas válidas de acuerdo con ella), metasistemáticas.

Bulygin está también de acuerdo con mi crítica a la propuesta de Nino de caracterizar «juez» en términos puramente fácticos y se muestra asimismo conforme con mi consideración de que, frente a la propuesta de MacCormick de definir «juez» exclusivamente en base a reglas sociales de deber, lo que define la posición normativa de «juez» no es sólo el hecho de que sobre quien la ocupa graviten determinados deberes -como el de juzgar y el de hacerlo en base a estándares preexistentes- sino también, y sobre todo, que quien ocupa tal posición sea titular del poder normativo de dictar determinaciones aplicativas de carácter obligatorio. Así pues, en opinión de Bulygin, una caracterización adecuada de «juez» sólo puede hacerse en términos normativos ( $\mathrm{y}$ no puramente fácticos) y haciendo referencia tanto a reglas que imponen deberes como a reglas que confieren poderes (y no sólo sobre la base de las primeras). Eso mismo pienso yo. $\mathrm{Y}$ es 
por ello -y ante la imposibilidad de caracterizar «juez», sin incurrir en circularidad, en términos de reglas jurídicas válidas de adjudicación- por lo que propongo una caracterización en términos de reglas sociales aceptadas que confieren poder jurisdiccional e imponen deberes relativos al ejercicio de dicho poder. Escribo así que «son jueces aquellos que son reconocidos socialmente como tales, esto es, como titulares de los deberes y poderes normativos que definen el rol judicial» y señalo que considero como deberes y poderes definitorios del rol judicial «el deber de decidir los casos que se presentan ante ellos, el deber de hacerlo sobre la base de estándares o reglas preexistentes y el poder de decidir tales casos con carácter obligatorio» (Ruiz Manero, 1990, pág. 133).

Esta propuesta es merecedora, a juicio de Bulygin, de diversas críticas. La primera de ellas es que -dice- «la teoría pasa por alto un dato empírico de suma importancia, a saber que- al menos en los órdenes jurídicos desarrollados o maduros- existen un gran número de normas legisladas (algunas de rango constitucional, otras legales) que regulan minuciosamente el status normativo de los jueces y que los juristas (y los simples mortales) usan esas normas para identificar a los jueces». No creo haber «pasado por alto» de ninguna manera el dato de que los ordenamientos jurídicos contienen, en efecto, numerosas y muy minuciosas reglas de adjudicación ni tampoco el que cotidianamente no tengamos dificultades especiales en determinar quiénes son los jueces de un determinado sistema jurídico. Me he limitado a poner de relieve que, si consideramos que el sistema jurídico está integrado por una regla última de reconocimiento aceptada por el conjunto de la judicatura y por las normas válidas de acuerdo con ella, necesitamos un criterio teórico general para identificar a los integrantes de dicha judicatura que no nos remita a normas cuya identificación presuponga la previa identificación de dichos integrantes.

La segunda crítica que me formula Bulygin es que «una teoría del Derecho que deja fuera del Derecho a gran número de normas que usualmente son consideradas normas jurídicas no puede considerarse satisfactoria». No veo ninguna razón por la que mi propuesta implique el dejar fuera del Derecho a un gran número de normas de adjudicación, que, desde luego, yo también considero normas jurídicas. Lo que sí implica, desde luego, es que si para identificar las normas jurídicas necesitarnos haber identificado previamente a los jueces, no podemos remitir a las normas jurídicas a la hora de trazar un criterio teórico de identificación de los jueces. 
Como tercera y última crítica señala Bulygin que «es fácil advertir que Ruiz Manero recurre, para escapar a la circularidad, a un simple truco verbal: llama "reglas sociales" a aquellas normas que determinan el status normativo del juez y que el resto de los mortales llama "normas jurídicas". Pero un cambio de nombre no es una solución satisfactoria para un problema conceptual». Como puede desprenderse de todo lo dicho antes, no creo haber recurrido a truco verbal alguno: me he limitado a proponer considerar la afirmación " $\mathrm{X}$ es juez en la sociedad Z" como sujeta a condiciones de verdad análogas a las que, según Hart, está sujeta la afirmación de que "en la sociedad Z existe el sistema jurídico S”. Como se sabe, Hart ha insistido en que "la afirmación de que un sistema jurídico existe es un enunciado bifronte, una de cuyas caras mira a la obediencia por parte de los ciudadanos ordinarios y la otra a la aceptación de reglas secundarias como pautas o criterios comunes críticos de conducta oficial, por parte de los funcionarios" (Hart, 1980, pág. 145). También la afirmación "X es juez en la sociedad Z" habría de considerarse, a mi juicio, «como un enunciado bifronte, cada una de cuyas caras hace referencia a una condición distinta: la primera, a que las decisiones dictadas por $\mathrm{X}$ sean, en general, efectivas (esto es, logren obediencia) y la segunda a que $\mathrm{X}$ sea reconocido, por parte de los aparatos (grupos organizados de personas) que poseen el monopolio de la fuerza en esa sociedad, como titular de los deberes y poderes que definen la posición institucional de "juez"» (Ruiz Manero, 1990, pág. 134).

Pero, en todo caso, todo esto no tendría mayor importancia si Bulygin tuviera razón en que resulta posible, sin incurrir en circularidad, construir un criterio teórico de identificación de los jueces en base a las normas jurídicas válidas de adjudicación.

Bulygin escribe que «todas estas dificultades desaparecen como por arte de magia una vez que reconozcamos que no hay deberes jurídicos que no sean establecidos por normas jurídicas y que para saber cuáles son las normas jurídicas tenemos que disponer de un criterio de identificación (una definición) de tales normas» y que por ello ha «propuesto que la regla de reconocimiento de Hart sea interpretada no como una norma que establece deberes a los jueces, sino como una regla conceptual que fija los criterios de identificación de un orden jurídico y de las normas que pertenecen a los diversos sistemas de ese orden»; «sólo habrá un orden jurídico -añade- cuando la población en general $\mathrm{y}$, sobre todo, los jueces y otros órganos oficiales compartan la misma definición» (subrayado mío).

Lamentablemente, no se ve por qué la consideración de la 
regla de reconocimiento como definición o regla conceptual y no como norma haya de presentar alguna ventaja en relación con el problema que ahora nos ocupa. Ello, a mi juicio, deja exactamente las cosas como estaban en la construcción de Hart. De acuerdo con la propuesta de Bulygin, llamaríamos «regla de reconocimiento» de un sistema jurídico al «criterio conceptual compartido» por los jueces y, en general, órganos oficiales, de dicho sistema y a su vez identificaríamos a los jueces y otros órganos oficiales de ese sistema en base a las normas identificadas como jurídicas sobre la base de ese criterio conceptual compartido por ellos. Tras la reconstrucción de Bulygin, el círculo nos sigue apareciendo, pues, tan redondo como lo encontrábamos en Hart. Si «desaparecen las dificultades» será, en efecto, «por arte de magia»: más exactamente, por un acto de fe, la cual, como es sabido, consiste en creer en lo que no se ve.

\section{BIBLIOGRAFÍA}

Bulygin, Eugenio ( 1976), «Sobre la regla de reconocimiento», en AAVV, Derecho, Filosofía y Lenguaje. Homenaje a Ambrosio L. Gioja, Astrea, Buenos Aires.

--- (1991), «Algunas consideraciones sobre los sistemas jurídicos». en este mismo número de Doxa. Hart, Herbert L. A. (1980), El concepto de Derecho, trad. de Genaro R. Carrió. Editora Nacional, México.

MacCormick, Neil (1981), H. L. A. Hart, Edward Arnold Publishers. Londres.

Nino, Carlos S. (1979), Algunos modelos metodológicos de «ciencia» jurídica, Universidad de Carabobo, Valencia (Venezuela).

--- (1980), Introducción al análisis del Derecho, $\quad$ Astrea, Buenos Aires.

--- (1985), La validez del Derecho, Astrea, Buenos Aires.

Raz, Joseph (1990), Practical Reason and Norms, 2. ed., Princeton University Press.

Ruiz Manero, Juan(1990), Jurisdicción y normas. Dos estudios sobre,función jurisdiccional y teoría del Derecho, Centro de Estudios Constitucionales, Madrid.

Ruiz Miguel, Alfonso (1988), «El principio de jerarquía normativa». en Revista Española de Derecho Constitucional, año 8 , núm. 24. 